\title{
METTL14 suppresses pyroptosis and diabetic cardiomyopathy by downregulating TINCR IncRNA
}

\author{
Liping Meng $\mathbb{D}^{1,3}$, Hui Lin $\mathbb{D}^{1,3}$, Xingxiao Huang ${ }^{1}$, Jingfan Weng ${ }^{1}$, Fang Peng $\mathbb{D}^{1 凶}$ and Shengjie Wu $\mathbb{D}^{2}{ }^{凶}$ \\ (c) The Author(s) 2021
}

N6-methyladenosine (m6A) is one of the most important epigenetic regulation of RNAs, such as IncRNAs. However, the underlying regulatory mechanism of $\mathrm{m} 6 \mathrm{~A}$ in diabetic cardiomyopathy (DCM) is very limited. In this study, we sought to define the role of METTL14-mediated m6A modification in pyroptosis and DCM progression. DCM rat model was established and qRT-PCR, western blot, and immunohistochemistry (IHC) were used to detect the expression of METTL14 and TINCR. Gain-and-loss functional experiments were performed to define the role of METTL14-TINCR-NLRP3 axis in pyroptosis and DCM. RNA pulldown and RNA immunoprecipitation (RIP) assays were carried out to verify the underlying interaction. Our results showed that pyroptosis was tightly involved in DCM progression. METTL14 was downregulated in cardiomyocytes and hear tissues of DCM rat tissues. Functionally, METTL14 suppressed pyroptosis and DCM via downregulating IncRNA TINCR, which further decreased the expression of key pyroptosis-related protein, NLRP3. Mechanistically, METTL14 increased m6A methylation level of TINCR gene, resulting in its downregulation. Moreover, the m6A reader protein YTHDF2 was essential for m6A methylation and mediated the degradation of TINCR. Finally, TINCR positively regulated NLRP3 by increasing its mRNA stability. To conclude, our work revealed the novel role of METTL14-mediated m6A methylation and IncRNA regulation in pyroptosis and DCM, which could help extend our understanding the epigenetic regulation of pyroptosis in DCM progression.

Cell Death and Disease (2022)13:38; https://doi.org/10.1038/s41419-021-04484-z

\section{BACKGROUND}

Diabetic cardiomyopathy (DCM), a major cardiovascular complication of diabetes, is characterized by myocardial fibrosis, ventricular remodeling, and cardiac dysfunction [1]. DCM is closely associated with the occurrence of heart failure, making it the majority cause of death among patients with diabetes [2]. DCM influences heart healthy through various mechanisms, including changes in metabolism, abnormal subcellular composition, and damage of microvascular [3]. However, the detailed mechanism of DCM is not well known and remains elusive. Revealing the key genes involved in DCM and identifying the potential regulatory mechanism will provide therapeutic targets used for overcoming DCM.

Pyroptosis is characterized by rapid plasma membrane rupture, with the consequent release of intracellular contents and proinflammatory mediators, such as caspase-1 [4]. The main signaling pathway involved in pyroptosis is mediated by caspase-1 activation, resulting in the maturation process of IL-1 $\beta, I L-18$, and gasdermin D (GSDMD) [5]. Previous studies widely reported the essential role of pyroptosis in cardiomyopathy, especially in DCM [6]. Xie et al. demonstrated that NLRP3 inflammasome mediated the chemerin/CMLR1-induced inflammation and pyroptosis and contribute to DCM [7]. Another research by Cao et al. revealed that high glucose-induced cardiotoxicity by inhibiting NLRP3 inflammasome activation and pyroptosis [8].
As the most abundant chemical modification of eukaryotic messenger RNA (mRNA), N6-methyladenosine (m6A) is known to influence various fundamental bioprocesses by regulating target gene expression [9]. $\mathrm{m6A}$ regulatory proteins are composed of the "erasers" FTO and ALKBH5, the "readers" YTHDFs and IGF2BPs, and the "writers" METTL3, METTL14 and WTAP [10]. Alterations in the m6A level mediate cell apoptosis, proliferation, self-renewal, and development [11]. However, the potential role of m6A methylation in DCM progression remains unknown.

Methyltransferase-like 14 (METTL14), a well-known m6A writer protein, widely participated in the progression of major diseases, such as cardiovascular pathogenesis [12]. However, whether METTL14 regulates DCM and the underlying mechanism are under investigation. In this study, we revealed that METTL14 was downregulated in myocardium tissues of DCM rat, and high glucose suppressed the METTL14 expression level. Mechanistically, the METTL14-mediated activity of m6A modification of TINCR suppressed pyroptosis of cardiomyocytes and DCM in an NLRP3dependent manner.

\section{MATERIALS AND METHODS}

\section{Cell lines and chemical reagent}

As we previously described [13], neonatal rat ventricular myocytes (NRVMs) were obtained from isolated heart tissues of young Wistar rats. Briefly, the

\footnotetext{
${ }^{1}$ Department of Cardiology, Shaoxing People's Hospital(Shaoxing Hospital, Zhejiang University School of Medicine), Shaoxing 312000 Zhejiang, China. ${ }^{2}$ Department of

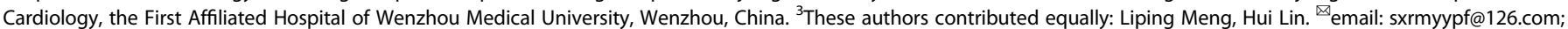
wusj120@163.com

Edited by Professor Sergio Lavandero
}

Received: 2 July 2021 Revised: 7 December 2021 Accepted: 17 December 2021

Published online: 10 January 2022 
dissected hearts minced in HEPES-buffered nic acid saline solution. Nonmyocyte contaminants were removed by two rounds of pre-plating for $1.5 \mathrm{~h}$ on $100-\mathrm{mm}$ plastic cell culture dishes under a culture condition of $37^{\circ} \mathrm{C}$ and $5 \% \mathrm{CO}_{2}$. Then, the cardiomyocytes were seeded into different culture dishes with medium containing serum. After incubation for 24 hours, medium without serum was used to replace the serumcontaining medium.

The normal H9c2 cardiomyocyte cell line was purchased from American Type Culture Collection (ATCC). Cells were cultured with 1640 medium containing $10 \%$ fetal bovine serum in a humidified atmosphere of $5 \% \mathrm{CO}_{2}$ at $37^{\circ} \mathrm{C}$. Actinomycin D was purchased from Sigma-Aldrich (St. Louis, MO, USA) and used at the concentration of $5 \mu \mathrm{g} / \mathrm{ml}$. MCC950 sodium (CP456773, CRID3 sodium salt), a NLRP3 inhibitor, was used at the concentration of $7.0 \mathrm{nM}$ in cell culture and $12 \mathrm{mg} / \mathrm{kg}$ in animal treatment (Selleck, Shanghai, China). For osmotic stress, hyperosmotic medium was made by adding various concentrations of D-sorbitol (Sigma-Aldrich) to regular 1640 medium for different durations. Standard 1640 culture condition was used as isosmotic media.

\section{Establishment and treatment of diabetic animal models}

A total of 120 Male Sprague-Dawley rats weighing 200-250 g (95-110 days old) were purchased from Model Animal Research Center of Nanjing University were randomly divided into respective treatment groups $(n=8$ per group). The diabetic model was constructed by a single intraperitoneal injection of streptozotocin $(65 \mathrm{mg} / \mathrm{kg})$, which imitates a model of type 1 diabetes. The fasting blood glucose was measured one week after injection. Only rats with glucose levels higher than $16.7 \mathrm{mmol} / \mathrm{L}$ were defined as diabetic. Cardiac function was investigated seven days following the last treatment, and the heart tissues were then isolated for expression analyses. The lentivirus vector used for silencing or overexpressing specific genes were dissolved in $50 \mu \mathrm{L}$ saline at the concentration of $1 \times 10^{9} \mathrm{TU}$ with one dose after the animal model was established. NLRP3 inhibitor MCC950 $(10 \mathrm{mg} / \mathrm{kg})$ was intraperitoneally injected $30 \mathrm{~min}$ before streptozotocin treatment. The experimental protocol was approved by IACUC of Shaoxing Hospital of Zhejiang University (Shaoxing city, China).

\section{RNA extraction and quantitative RT-PCR}

Total RNA was isolated by TRlzol reagent (Invitrogen, Carlsbad, CA). A total of $1 \mathrm{mg}$ of extracted RNA was used to perform reverse transcription using Superscript III transcriptase (Invitrogen). Quantitative real-time PCR (qRTPCR) was performed using SYBR Green real-time PCR analysis using BioRad CFX96 system (Bio-Rad, Cambridge, MA) with the specific primers. Expression of the PCR data were shown as $2^{-\Delta \Delta c t}$, and normalized to the internal control level (GAPDH).

\section{Echocardiography}

Rats from respective treatment groups were fixed on operating table and were performed under sodium pentobarbital anesthesia $(75 \mathrm{mg} / \mathrm{kg})$, and all efforts were made to minimize suffering. Echocardiography was performed using a Vivid 7 Dimension (GE Healthcare, Munich, Germany) echocardiograph equipped with a 14-MHz transducer. Parasternal shortaxis views were used for M-mode analysis. Enddiastolic and endsystolic left ventricular inner diameters (LVIDd, LVIDs) were measured, and fractional shortening (FS) and left ventricular ejection fraction (LVEF) were calculated

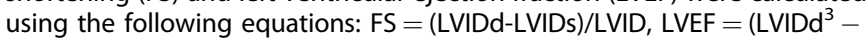
$\left.\mathrm{LVIDs}^{3}\right) / \mathrm{LVIDd}^{3}$.

\section{Transmission electron microscopy}

Hearts were extracted, cut finely into small $\left(\sim 1 \mathrm{~mm}^{3}\right)$ blocks, and fixed overnight in $4 \%$ glutaraldehyde in $100 \mathrm{mM}$ phosphate buffer, followed by post-fixation in $2 \%$ osmium tetroxide in $100 \mathrm{mM}$ phosphate buffer. Specimens then underwent en bloc treatment with uranyl acetate, dehydration in ethanol, and transferred to propylene oxide, prior to embedding. Ultra-thin sections $(50-70 \mathrm{~nm})$ were cut and stained with uranyl acetate and lead citrate, and examined in a JEOL 1200EX electron microscope.

\section{TUNEL assay}

Cells were firstly fixed with $4 \%$ paraformaldehyde for $30 \mathrm{~min}$ followed by staining with one-step TUNEL kit as per the instructions of the manufacture (Beyotime, Shanghai, China). The positive stained parts were visualized and calculated via a fluorescence microscopy (Axio Observer A1, ZEISS, Germany).

\section{IHC analysis}

Heart myocardial tissues were isolated and fixed with 4\% Paraformaldehyde followed with incubation with primary antibodies against respective proteins: NRLP3 (1:100, cat. no. ab214185, Abcam, Cambridge, MA), METTL14 (1:100, ab223090, Abcam), cleaved caspase-1 (1:100, ab1872, Abcam) and GSDMD-N (1:100, bs-14287R, Bioss, Beijing, China), to detect their expression levels. Images were visualized using a ZEISS Axio Observer A1 (Oberkochen, Germany) microscope system and processed with ZEISS software.

\section{Masson staining}

After isolation of rat heart from respective treatment groups, the tissues were fixed with $4 \%$ paraformaldehyde followed by embedding with paraffin and sectioning. Masson staining process was done by using Masson's Trichrome Stain Kit as per the guidelines of manufacture (cat.no. G1340, Solarbio, Beijing, China). The sections were treated sequentially with hematoxylin and ferric oxide, acid fuchsin, phosphomolybdic acid, and acetic acid. The quantification was performed with Image $J$ following mounting of the sections with neutral gum.

\section{RIP and RNA pulldown assay}

The RNA immunoprecipitation (RIP) was performed using the EZ-Magna RIP kit (Millipore, Burlington, MA, USA) according to the manufacturer's instructions. Briefly, $10^{7}$ cells were lysed with RIP lysis buffer using one freeze-thaw cycle. Cell extracts were coimmunoprecipitated with anti-m6A (ab208577, Abcam) and NLRP3 (ab263899, Abcam) antibodies and the retrieved RNA was subjected to qRT-PCR analysis.

The RNA pulldown assay was performed using a Magnetic RNA-Protein Pull-down Kit (Thermo Scientific) according to the manufacturer's instructions. The 3'-end Biotin-TEG modified-DNA probes against TINCR were synthesized by Sangon (Shanghai, China). The cell lysates were hybridized with a mixture of biotinylated DNA probes for $4 \mathrm{~h}$ at $37^{\circ} \mathrm{C}$. The binding complexes were then recovered using streptavidin-conjugated magnetic beads. Finally, protein was eluted and purified from the beads for western blot analyses.

\section{Western blots}

Protein extraction was performed using RIPA lysis buffer (Pierce, IL, USA) containing protease inhibitor (Roche, CA, USA). Protein extracts were subjected to $10 \%$ SDS-polyacrylamide gel electrophoresis followed by electro-transfer to polyvinylidene difluoride membrane. After $1 \mathrm{~h}$ of premembrane blocking with 5\% BSA, the proteins were incubated with respective primary antibodies at $4{ }^{\circ} \mathrm{C}$ overnight followed by secondary antibodies incubation at room temperature for $1 \mathrm{~h}$. The detection of proteins was carried out using ECL reagent. Primary antibodies used in this study include: NRLP3 (1:1000, cat. no. ab214185, Abcam), METTL14 (1:1000, ab223090, Abcam), cleaved caspase-1 (1:1000, ab1872, Abcam), IL-1 $\beta$ (1:1000, ab2105, Abcam) and IL-18 (1:1000, ab18672, Abcam), GSDMD-N (1:1000, bs-14287R, Bioss, Beijing, China) and GAPDH (Invitrogen, cat. no. PA1-987).

\section{Statistical analysis}

All experiments were performed in triplicate. Statistics were presented as mean \pm SD. Comparison between two groups was analyzed using the Student's $t$-test. Fisher exact testing was performed to evaluate the difference of proportions between different groups. Statistical analyses were performed using GraphPad Prism (v8.0.1, GraphPad Software Inc., San Diego, CA, USA). $P<0.05$ was considered to indicate a statistically significant difference.

\section{RESULTS}

\section{Pyroptosis is involved in DCM through NLRP3-caspase-1} pathway in vitro

We treated neonatal rat ventricular myocytes (NRVMs) and cardiomyocyte cell line $\mathrm{H} 9 \mathrm{c} 2$ with glucose at the concentration of $5.5 \mathrm{mmol} / \mathrm{L}$ (normal control) or $50 \mathrm{mmol} / \mathrm{L}$ (high glucose, $\mathrm{HG}$ ) to imitate the hyperglycemic condition. HG status was generated 
A

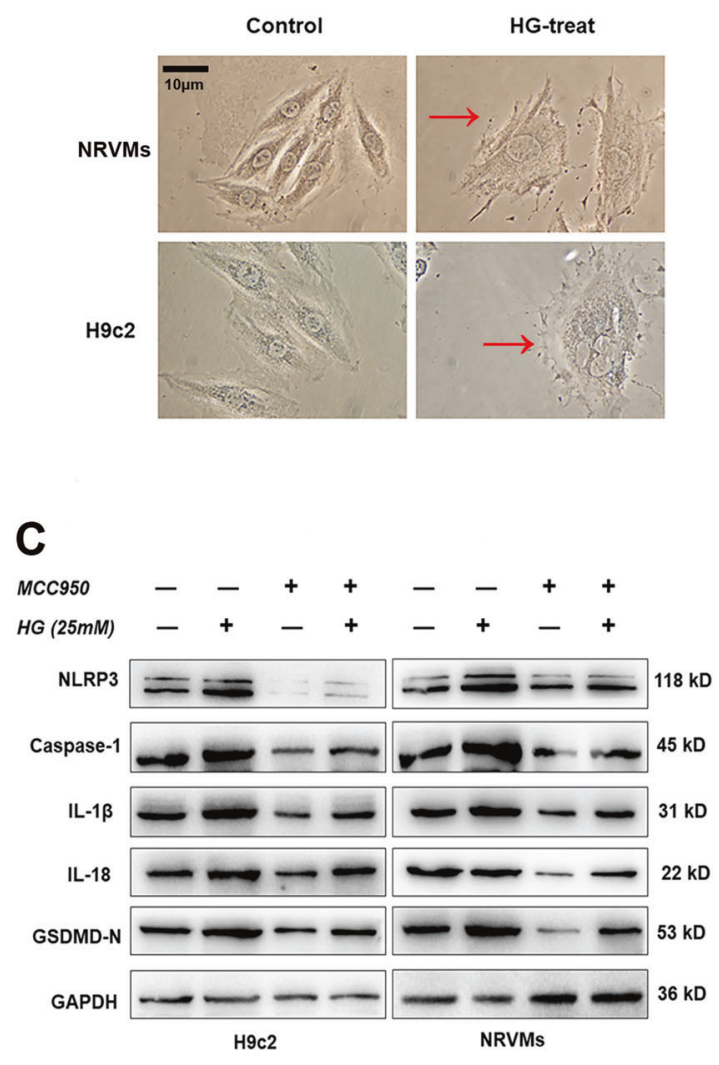

B
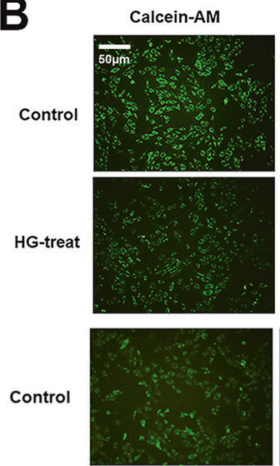

HG-treat

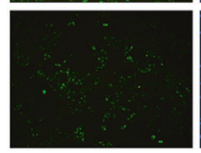

D

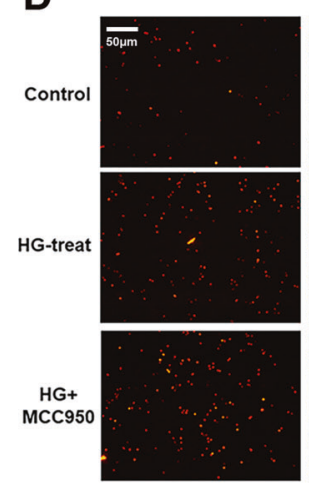

DAPI
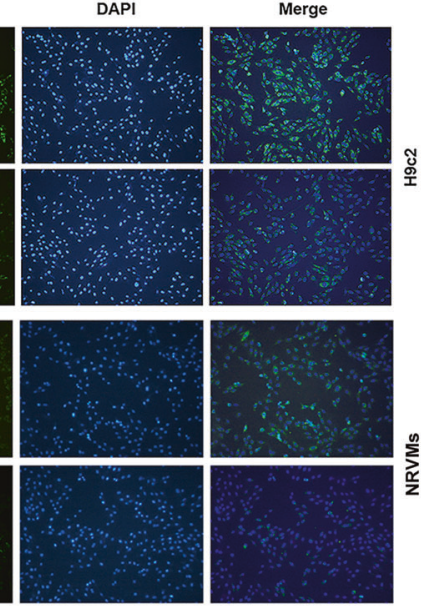

H9c2
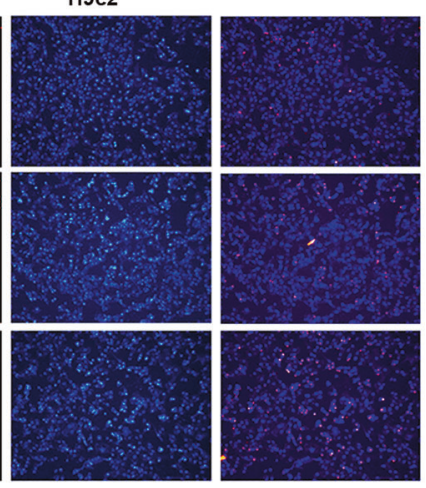

Fig. 1 HG treatment induces pyroptosis through targeting NLRP3. A Morphologic changes of cells cultured with HG were consistent with pyroptosis, including swelling changes and rupture of cell membrane. B Calcein-AM (dyed with green) staining of HG-treated and controlled cardiomyocytes were shown. C Western blots were performed to detect the expression level of pyroptosis-related proteins in cardiomyocytes treated with HG and/or NLRP3 inhibitor MCC950 (5 $\mathrm{gg} / \mathrm{ml})$ for $24 \mathrm{~h}$. D TUNEL staining was used for evaluating the apoptosis level of H9c2 cells treated with HG and(or) MCC950 (10 mg/kg).

with the concentration of $50 \mathrm{mmol} / \mathrm{L}$ since another reported concentration used in $\mathrm{HG}$ was not enough for activating pyroptosis, such as $25 \mathrm{mmol} / \mathrm{L}$ (Supplementary Fig. S1A). As shown in Fig. $1 A$, due to the HG treatment, H9c2 cells exhibited characteristic morphological changes, such as swelling changes and rupture of the cell membrane, suggesting that pyroptosis was directly involved in DCM process. Then we performed Calcein-AM staining analysis and found that HG treatment caused an increased damage of cell membrane when compared with control treatment (Fig. 1B and Supplementary Fig. S1B). Moreover, the protein expression of pyroptosis markers, including NLRP3, cleaved caspase-1, and GSDMD-N, were significantly upregulated in HG-treated cardiomyocytes. Importantly, treatment with MCC950, a well-known NLRP3 inhibitor, suppressed pyroptosisrelated proteins and dramatically reversed pyroptosis of cells treated with HG (Fig. 1C and Supplementary Fig. S1C). We also evaluated the effect of HG treatment on cell apoptosis by performing TUNEL staining. As shown, apoptosis was activated upon HG treatment, however, this activation was not restored by MCC950 treatment in H9c2 cells (Fig. 1D and Supplementary Fig. S1D). For in vivo data, IHC showed a decreased expression of NLRP3 upon MCC950 treatment (Supplementary Fig. S1E). However, echocardiography and ELISA showed that MCC950 showed no effect on cardiac function and inflammation (Supplementary Fig. S1F, G). Finally, we treated H9c2 cells with D-sorbitol at different concentrations, representing hyperosmotic pressures, to verify the effects of hyperosmotic condition on pyroptosis. As shown, pyroptosis-related proteins were not activated under sole hyperosmotic conditions (Supplementary Fig. $\mathrm{S} 1 \mathrm{H})$, which excluded the involvement of hyperosmotic pressure during HG treatment-induced pyroptosis. These data showed that HG treatment resulted in pyroptosis of cardiomyocytes in an NLRP3-dependent manner.

\section{Pyroptosis was activated in cardiomyocyte of DCM rats}

To further verify the essential role of pyroptosis in HG-induced toxicity of cardiomyocytes, we constructed DCM model using Wistar rats by single intraperitoneal injection of streptozotocin, an animal model that mimics type 1 diabetes. Echocardiography was performed to evaluate the cardiac function of DCM rats. Our data revealed a reduced LVEF and FS in rats with DCM in contrast to normal rats. The LVEF level of DCM rats was $48 \%$, which is significantly lower than that in normal rats (68\%). Moreover, the average FS of rats with DCM was also dramatically decreased compared to control rats ( $21 \%$ vs. $37 \%$ ) (Fig. 2A). Interestingly, when NLRP3 was silenced with MCC950 in DCM rats, the damaged cardiac function caused by DCM was dramatically reversed (Fig. 2A). By detecting myocardial enzyme markers, such as AST, LDH, and $C K-M B$, we identified an elevated expression in DCM rats, however, MCC950 treatment partly restored this effect. (Fig. 2B). In addition, electron microscopy imaging of cardiomyocyte ultrastructure showed that DCM rats showed serious cardiomyocyte damage compared to control hearts, including increased intermitochondrial distance, disconnected cardiac myofibers, and thinner myofibers, and this damage could be partially rescued by MCC950 (Fig. 2C). Finally, the expressions of pyroptosis 


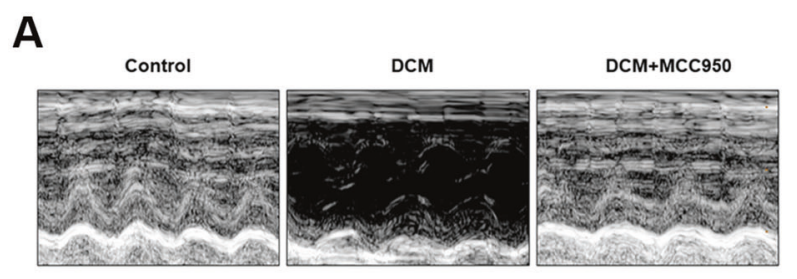

B
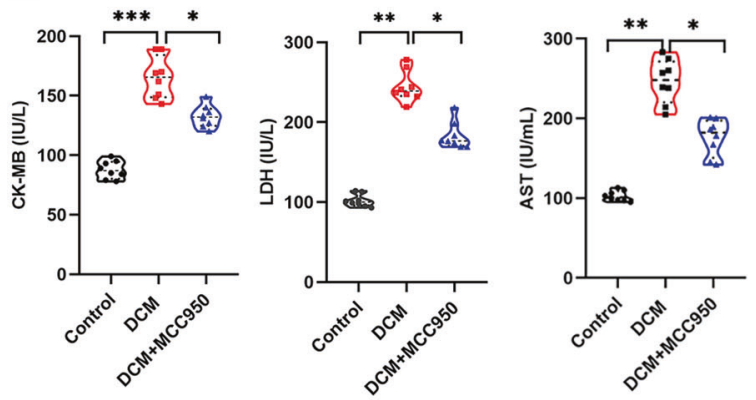

D

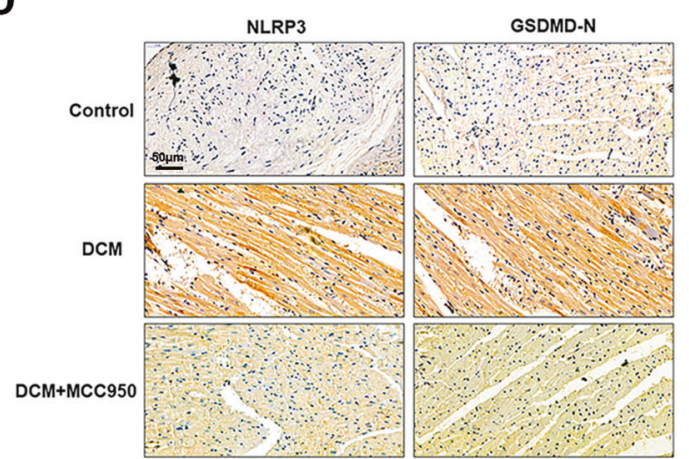

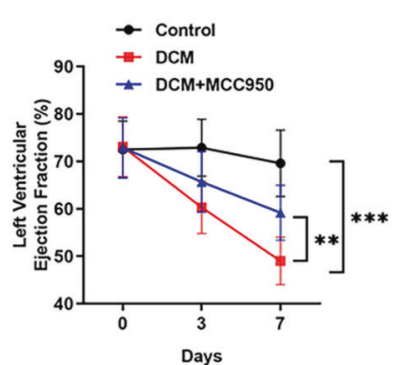

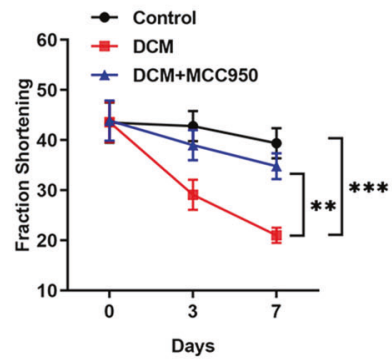

C

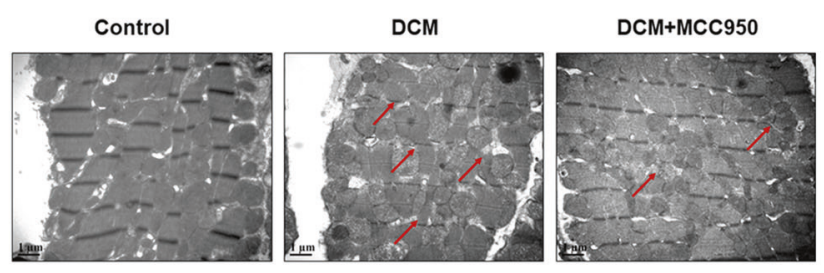

E

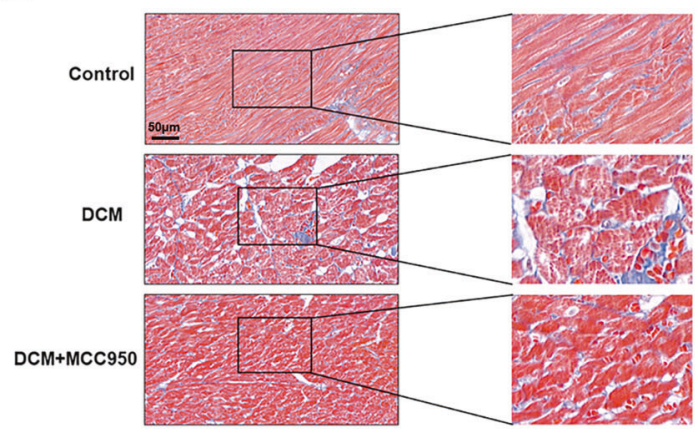

Fig. 2 Pyroptosis was involved in DCM in vivo. A Morphological changes (left panel) and quantitative analysis of LVEF and FS (right panel) in rat hearts were detected by echocardiography, ${ }^{* *} P<0.01,{ }^{* *} P<0.001$. B Serum expression of myocardial enzymes CKMB, LDH and AST were quantified in rats of respective groups, ${ }^{*} P<0.05$, ${ }^{* *} P<0.01,{ }^{* * *} P<0.001$. C A representative section of the left ventricle from respective groups of rats showing sporadic mitochondrial, damaged disconnected myofibers, and thinner myofibers. D IHC analysis was performed to detect the expression of NLRP3 and GSDMD-N in hearts of DCM rat after treated with MCC950. E Masson staining was done in heart tissues of DCM rats treated with MCC950.

proteins, NLRP3 and GSDMD-N, were dramatically increased in rats of DCM compared, meanwhile, this influence was significantly relieved by MCC950 (Fig. 2D). Since pyroptosis is accompanied with rupture of cardiomyocyte membrane, causing the damage and disruption of collagens, thus the collagen status could reflect the process of pyroptosis. Masson staining showed that collagen was damaged in DCM rats and further reversed by MCC950 treatment (Fig. 2E). Taken together, we demonstrated that DCM was closely associated with cardiomyocytes pyroptosis in an NLRP3-dependent manner.

\section{METTL14 is downregulated in DCM}

To find whether METTL family was involved in pyroptosis in DCM models, we detected the expression level of METTL14 and METTL3 in DCM rats. As shown in Fig. 3A, B, METTL14 was significantly downregulated in heart tissue and serum samples of DCM rats compared to those of controlled rats. Moreover, a decreased METTL14 level was identified in NRVMs and H9c2 cells treated with HG when compared with control cells (Fig. 3C, D). Specially, METTL14 level was not dysregulated under hyperosmotic pressures with D-sorbitol treatment (Supplementary Fig. S2A). On the other hand, METTL3 expression was not altered according to our in vitro and in vivo data (Supplementary Fig. S2B, C). These indicate that METTL14 may be a key molecular regulator during DCM initiation and progression.

\section{METTL14 suppresses DCM via modulating pyroptosis}

To clarify the role of $\mathrm{m} 6 \mathrm{~A}$ modification in pyroptosis and DCM progression, we evaluated the effect of METTL14 in DCM progression. As expected, injection of LV-METTL14 into DCM rats significantly increased m6A level in DCM rats (Fig. 4A, B). Echocardiography suggested that overexpression of METTL14 increased LVEF, FS (Fig. 4C). Meanwhile, electron microscopy revealed a relieved injury in cardiomyocyte (Fig. 4D). Moreover, enhanced METTL14 inhibited pyroptosis level in myocardial tissues, including downregulation of NLRP3, caspase-1, and GSDMD-N (Fig. 4E), suggesting that METTL14-mediated m6A modification may suppress DCM through modulating pyroptosis. To confirm this hypothesis in vitro, we silenced METTL14 and m6A modification level in H9c2 and NRVMs cells (Fig. 4F, G). Reversed morphologic changes of pyroptosis were observed upon the silence of METTL14 (Fig. 4H). Western blotting showed that shMETTL14 caused upregulated NLRP3, cleaved caspase-1, and GSDMD-N (Fig. 4I). Consistently, Calcein-AM staining revealed an increased membrane damage in $\mathrm{H} 9 \mathrm{c} 2$ cells silenced with METTL14 compared to controls, however, this effect was abrogated by 


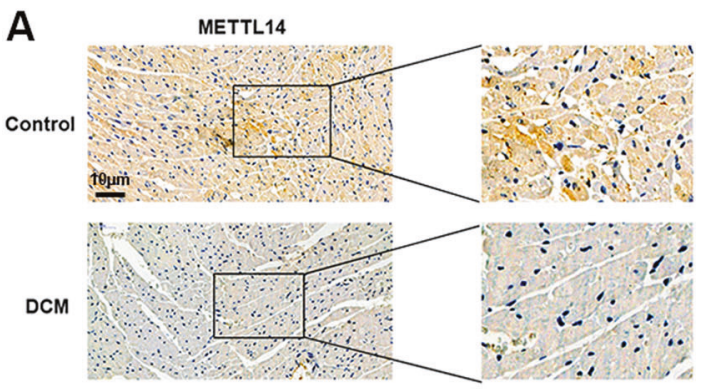

B
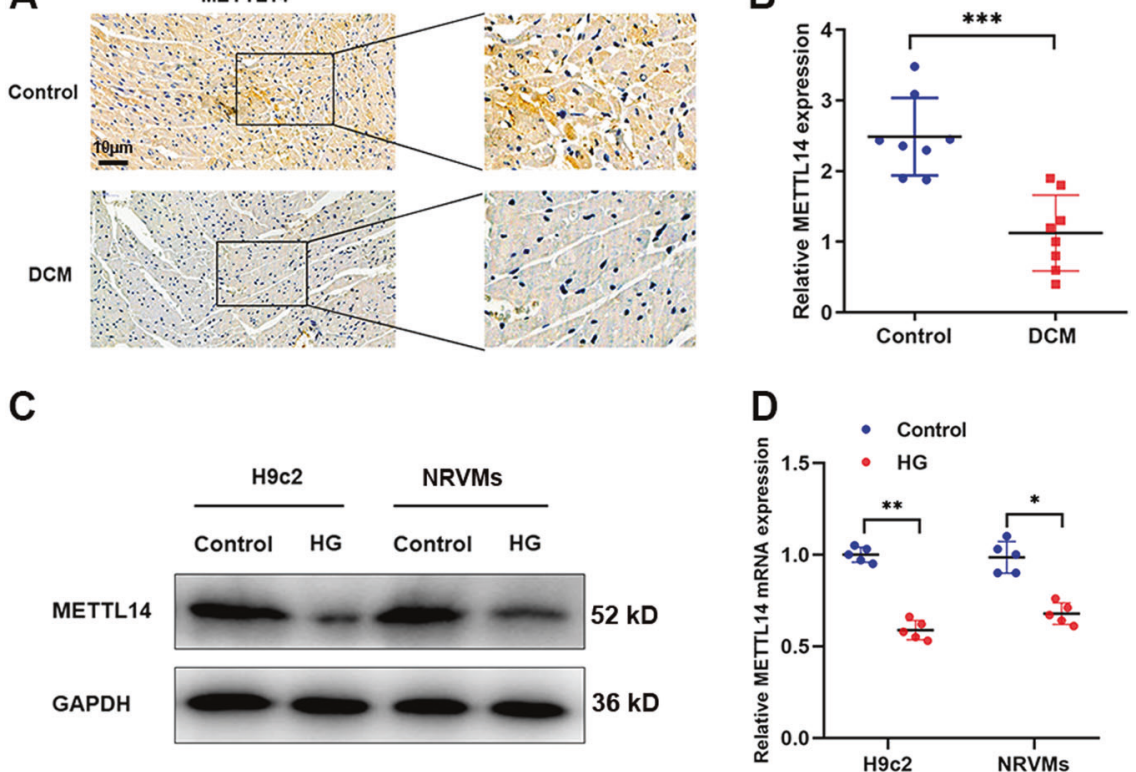

Fig. 3 METTL14 was downregulated in DCM rats. A METTL14 protein expression was detected in heart tissues of DCM and control rats. B Serum circulating METTL14 mRNA was determined in rats of respective groups, ${ }^{* *} P<0.001$. C, D METTL14 protein (C) and mRNA (D) levels were detected in cardiomyocytes treated with $\mathrm{HG},{ }^{*} P<0.05,{ }^{* *} P<0.01$.

MCC950 (Fig. 4J). Collectively, we proved that METTL14-mediated $\mathrm{m} 6 \mathrm{~A}$ modification plays essential roles in DCM via regulating cardiomyocyte pyroptosis.

\section{METTL14 suppresses pyroptosis via targeting TINCR IncRNA}

It is reported that $\mathrm{m} 6 \mathrm{~A}$ process was closely associated with IncRNA processing [14]. By performing GSEA analysis, we revealed that METTL14 was involved in the process and degradation of ncRNAs (Fig. 5A). Based on our previous observation of IncRNA TINCR in pyroptosis, we supposed that METTL14 may regulate DCM through TINCR-mediated pyroptosis. To prove this assumption, we detected TINCR expression and found that TINCR was negatively correlated with METTL14 in patients with DCM (Fig. 5B). In addition, TINCR was upregulated in HG-treated cells and DCM rats (Fig. 5C, D). Next, we silenced METTL14 in NRVMs and $\mathrm{H} 9 \mathrm{C} 2$ cells, and found that TINCR was upregulated accordingly (Fig. 5E), while overexpression of METTL14 leads to a decreased expression of TINCR (Fig. 5F).

Then, we performed gain-or-loss functional assays by injection of TINCR lentiviral vector into METTL14-overexpressing DCM rats. Intriguingly, overexpression of TINCR reversed the METTL14induced effects on LVEF, FS, and cardiomyocyte damage in DCM rats (Fig. 5G, H). In addition, enhanced TINCR abrogated METTL14caused suppression of pyroptosis in H9c2 and NRVMs (Fig. 5I). Meanwhile, suppression of TINCR rescued pyroptosis caused by METTL14 silencing in normal rats as evidenced by western blots, electron microscopy imaging, and echocardiography analyses (Supplementary Fig. S3A-C). Collectively, we proved that IncRNA TINCR was, at least partly, responsible for METTL14-induced suppression of pyroptosis and DCM.

\section{METTL14-dependent m6A methylation downregulated expression of TINCR}

Take a step further, we sought to find whether it is the METTL14mediated m6A modification that downregulated TINCR expression. By analyzing the potential m6A binding sites with online SRAMP database (http://www.cuilab.cn/sramp), we verified 45 m6A residues located across TINCR sequence (Fig. 6A), among which 11 were identified as high/very high confidence (Table 1). It is well demonstrated that METTL14 acted as an interactor with
WTAP, binding to the methyltransferase to form a complex which mediates m6A methylation on RNAs. Here, sh-WATP vector was injected into METTL14-overexpressed DCM rats (Fig. 6B). Suppressed TINCR caused by METTL14 was abrogated by silence of WATP (Fig. 6C). Then, we performed RIP assay using m6A antibody, and found a significantly decreased methylated TINCR (site 7709) bounded by m6A upon METTL14 deletion or WTAP deletion (Fig. 6D). The above results strongly suggest that METTL14-dependent $\mathrm{m} 6 \mathrm{~A}$ modification of TINCR results in its downregulation.

m6A methylation is a characterized process that needs the involvement of $\mathrm{m} 6 \mathrm{~A}$ reader proteins, including YTHDFs [15]. Given that YTHDF2 participated in the modulation of m6A-dependent RNA degradation [16], we, therefore, assumed that YTHDF2 may be essential for METTL14-mediated TINCR decay and downregulation. By performing RIP using antibody against YTHDF2, we identified the positive binding of TINCR by YTHDF2 antibody in DCM rats (Fig. 6E). Consistently, RNA pulldown assay showed that YTHDF2 was significantly enriched by TINCR (Fig. 6F). Then, we silenced YTHDF2 expression in cardiomyocytes (Fig. 6G). The decay rate of TINCR was significantly slower in shYTHDF2-infected cells (Fig. 6H), indicating that YTHDF2 mediated m6A-RNA decay of TINCR. Altogether, our results revealed that METTL14-mediated m6A modification inhibited TINCR expression via YTHDF2regulated RNA degradation.

\section{TINCR promotes pyroptosis and DCM via stabilizing NLRP3 mRNA}

Previously, we demonstrated that TINCR regulated cardiomyocytes pyroptosis via stabilizing NRLP3 in DOX-induced cardiotoxicity [13]. To verify whether this regulation mode applies in DCM, we performed gain-and-loss functional assays. As shown, MCC950 remarkedly abrogated the TINCR-regulated pyroptosis in both HG-treated untreated cardiomyocytes (Fig. 7A and Supplementary Fig. S4). To further confirm the direct interaction between TINCR and NLRP3 RNA, we conducted RNA pulldown assay by generating biotinylated oligonucleotides in HG-treated H9c2 and NRVMs cells. As shown, NLRP3 RNA was remarkably enriched by biotinylated TINCR (Fig. 7B). By treatment with actinomycin D (ActD), a well-known inhibitor for RNA 


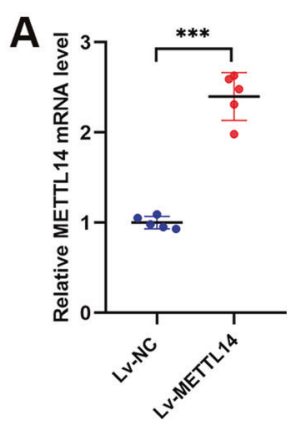

D
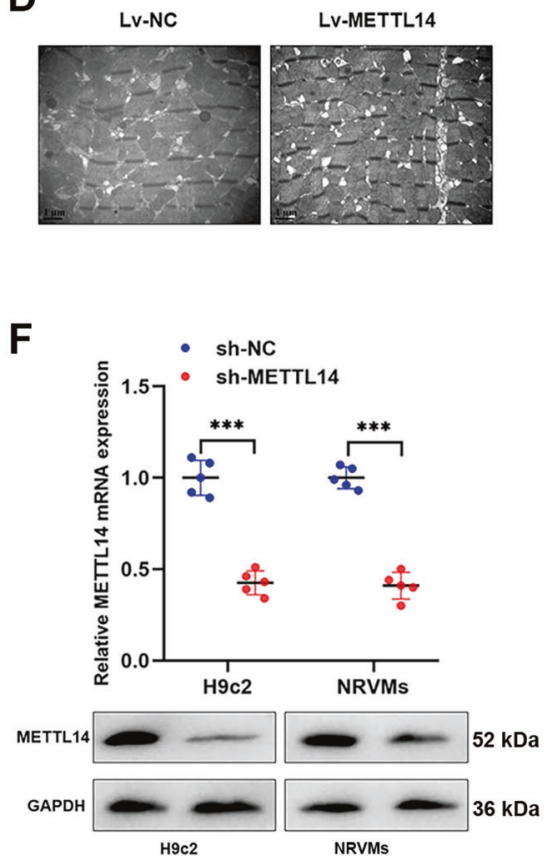

I

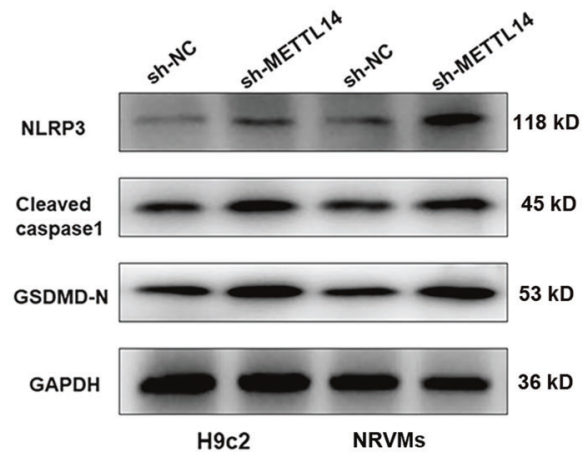

B

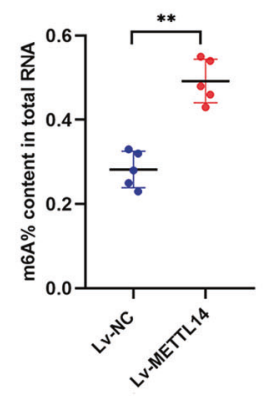

E

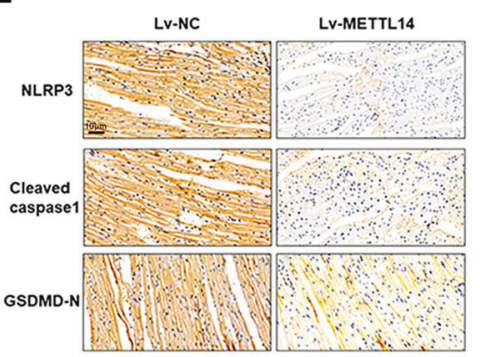

C
Lv-NC
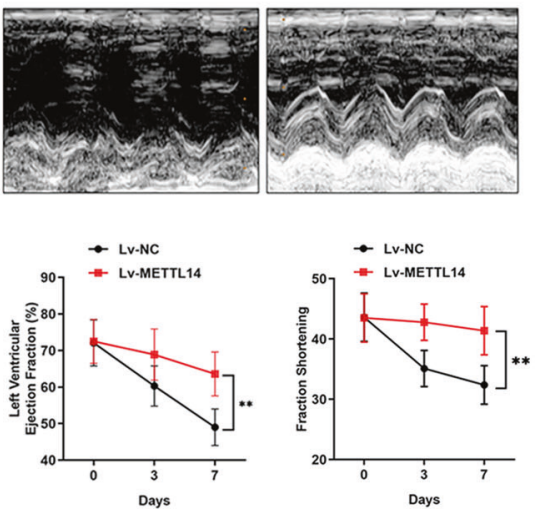

Lv-METTL14

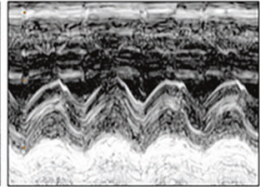

H

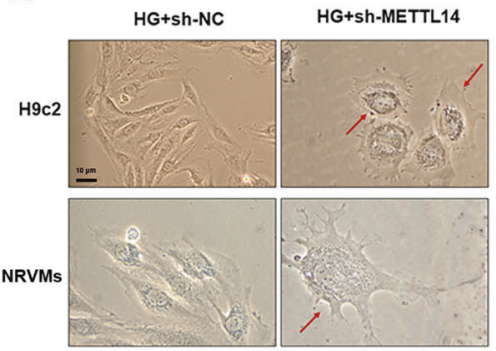

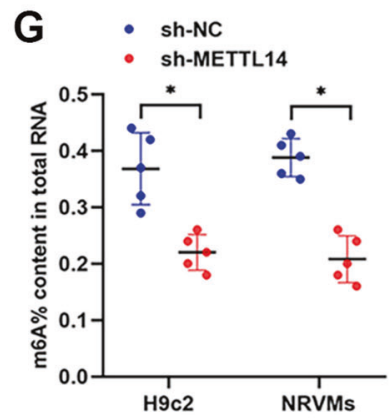

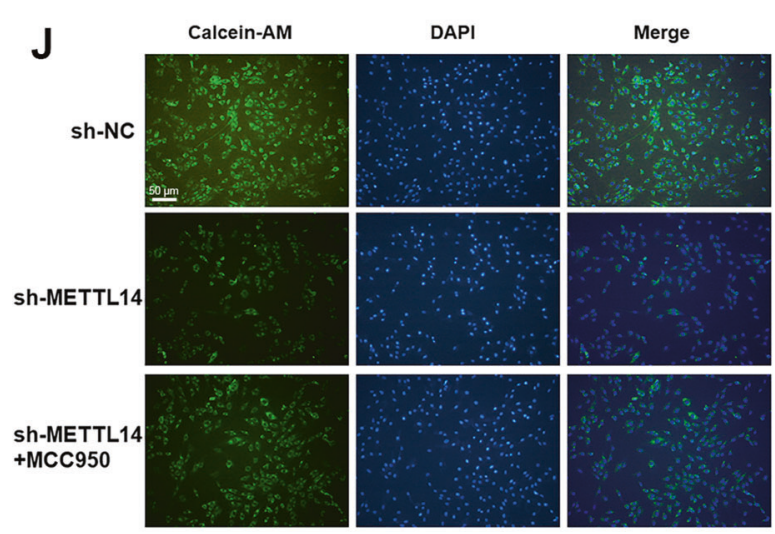

Fig. 4 METTL14 suppresses DCM via modulating pyroptosis. A Validation of METTL14 overexpression in DCM rats by western blot and qRT$\mathrm{PCR},{ }^{* * *} P<0.001$. B The overall m6A content was increased by upregulation of METTL14, ${ }^{* *} P<0.01$. C Morphological changes (upper panel) and quantitative analysis of LVEF and FS (lower panel) in rat hearts were detected by echocardiography after injection of METTL14 overexpression vector, ${ }^{* *} P<0.01$. D Electron microscopy imaging of cardiomyocyte ultrastructure showed that rats overexpressed with METTL14 showed decreased cardiomyocyte damage compared to control group. E IHC analysis of pyroptosis-related proteins in DCM rats overexpressed METTL14. F Confirmation of silence of METTL14 in cardiomyocytes at both transcript and protein levels, ${ }^{* * *} P<0.001$. G knockdown of METTL14 caused significantly downregulated m6A modification level, ${ }^{*} P<0.05$. $\mathbf{H}$ Morphologic changes of HG-treated cardiomyocytes upon knockdown of METTL14. I Western blot experiment was carried out to reveal the expression changes of pyroptosisrelated proteins in cardiomyocytes transfected with METTL14 silencing vectors. J Calcein-AM staining showed that deletion of METTL14 increased membrane rupture of cardiomyocytes, however, this effect was reversed by treatment with MCC950. 
A

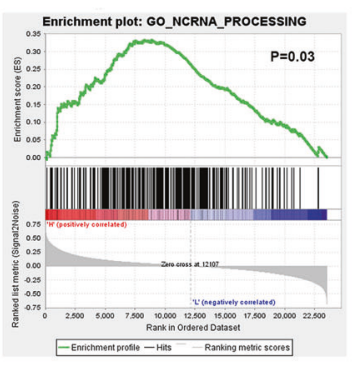

D

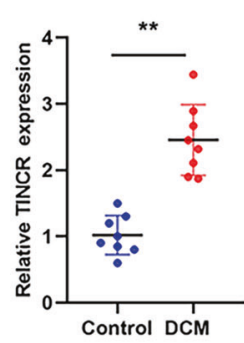

B

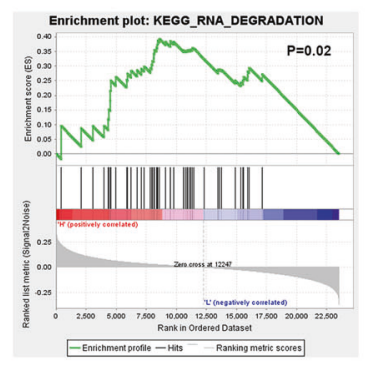

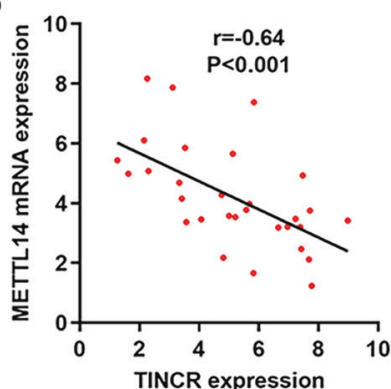

C

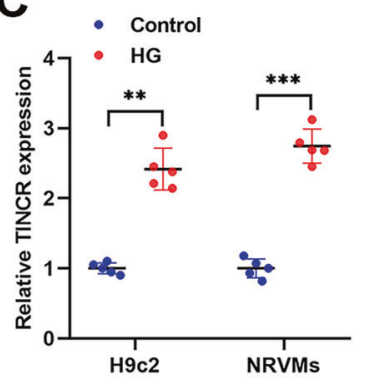

E : sh-NC

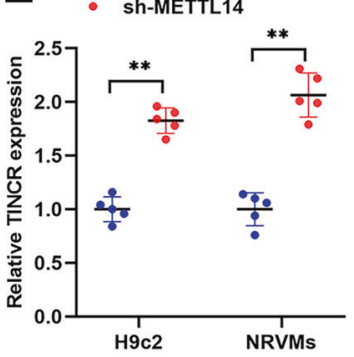

$F$ - LV-NC

- Lv-METTL14

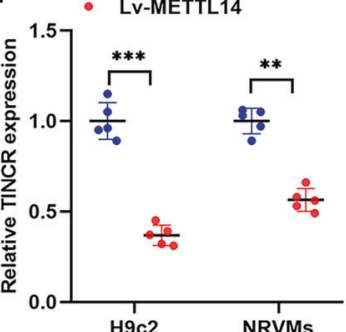

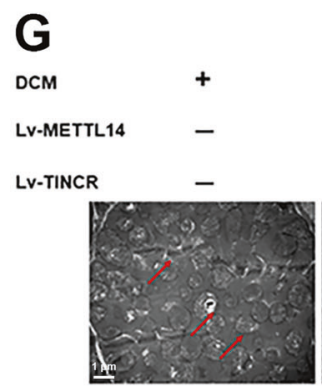
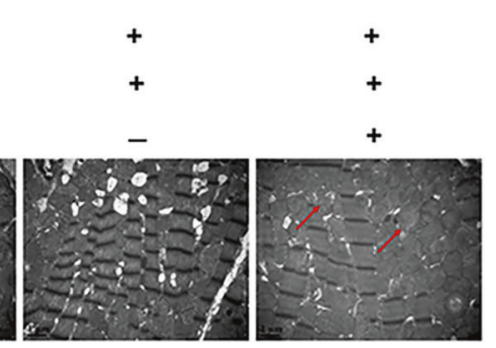

$\mathrm{H}_{\mathrm{ocm}}$
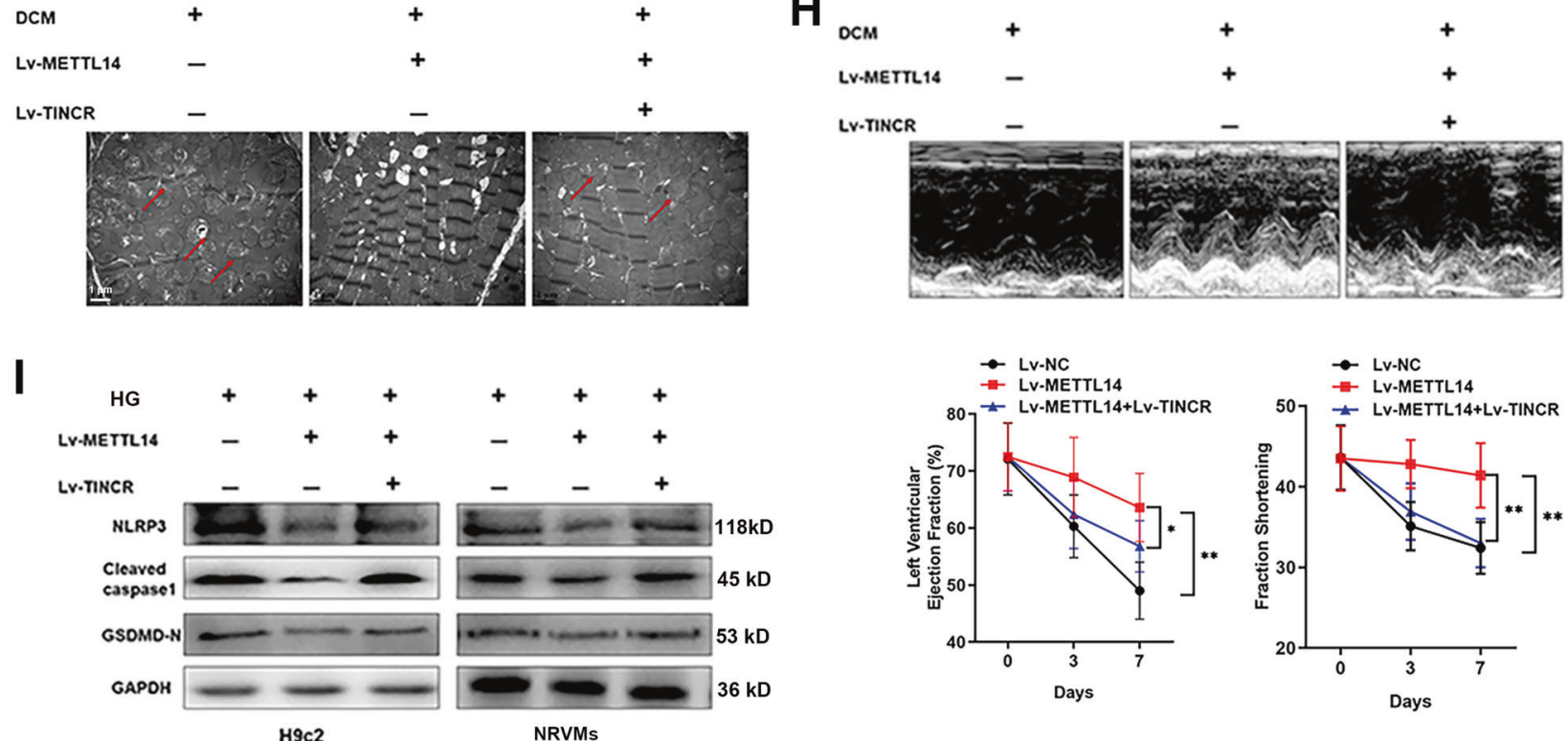

Fig. 5 METTL14 suppresses pyroptosis via targeting TINCR IncRNA. A GSEA analysis revealed a positive correlation between METTL14 and ncRNA processing and degradation. B A significant negative association was identified between METTL14 mRNA and TINCR expression. C HG treatment of cardiomyocytes significantly increased TINCR expression, ${ }^{* *} P<0.01,{ }^{* *} P<0.001$. D. TINCR was significantly upregulated in DCM rats in contrast to controlled rats, ${ }^{* *} P<0.01$. E, F qRT-PCR showed that silence of METTL14 (E) upregulated, while overexpression of METTL14 (F) suppressed TINCR expression in cardiomyocytes, ${ }^{* * P}<0.01$. G, $\mathbf{H}$ Cardiac function analysis $(\mathbf{G})$, electron microscopy imaging $(\mathbf{H})$ showed that injection of METTL14 dramatically reversed the myocardial damage caused by DCM, however, co-expression of TINCR abrogated the METTL14-mediated influence, ${ }^{*} P<0.05$, ${ }^{* *} P<0.01$. I The expressions of pyroptosis-related proteins was detected in H9c2 and NRVMs cells upon indicated treatments.

transcription, we evaluated the effect of TINCR on downstream mRNA degradation. The results revealed that knockdown of TINCR resulted in accelerated degradation of NLRP3 mRNA in cardiomyocytes (Fig. 7C). More importantly, METTL14 decreased the stability of NLRP3, and overexpression of TINCR reversed this effect (Fig. 7D). Collectively, the above results strongly support that METTL14-mediated m6A induced suppression of TINCR, which further regulates pyroptosis via stabilizing NLRP3 mRNA (Fig. 7E).

\section{DISCUSSION}

It is estimated that DCM occurs in approximately $12 \%$ of diabetic patients [17]. Clinical data suggest that DCM is closely associated with Clinical studies that indicate that DCM increases the risk of overt heart failure and induces worse prognosis in diabetic patients [18]. A strategy for prevention and treatment in order to improve the prognosis of DCM has not been established. In this study, we established that METTL14, a well-known m6A writer, inhibited pyroptosis and DCM through downregulation of IncRNA 
A

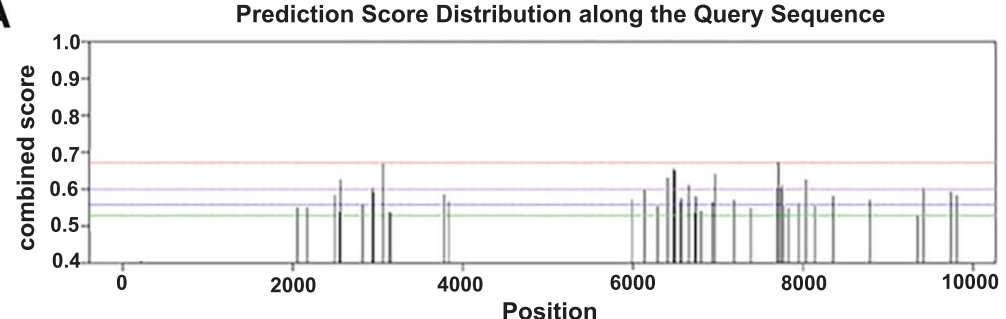

B

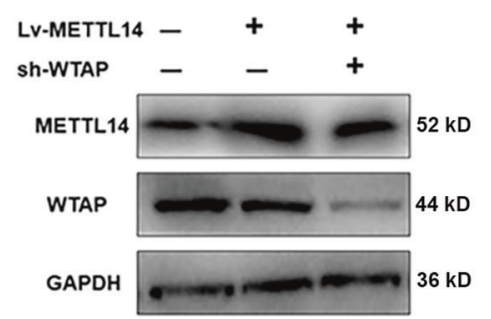

C

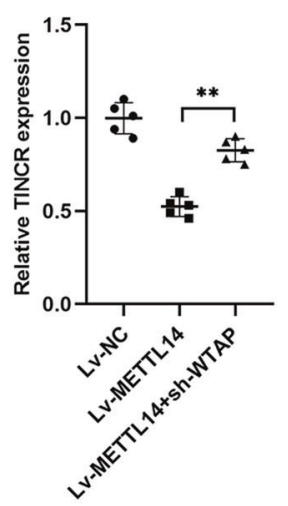

G

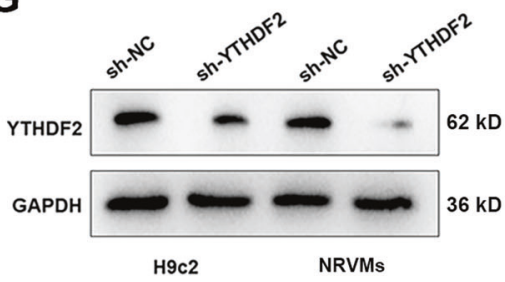

E
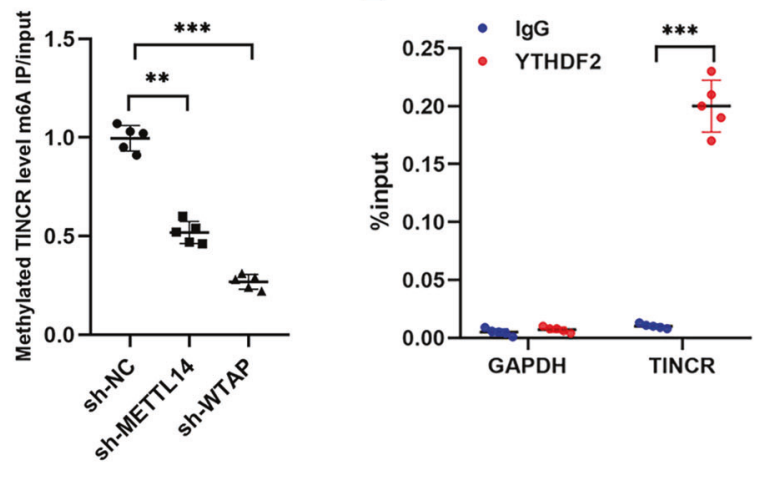

F

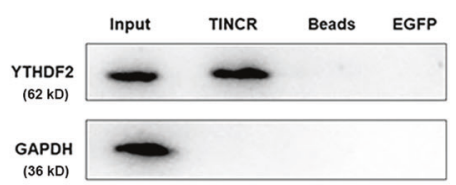

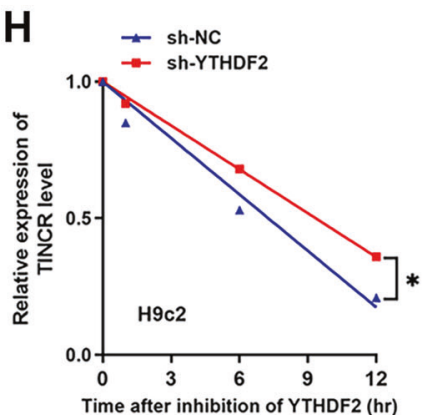

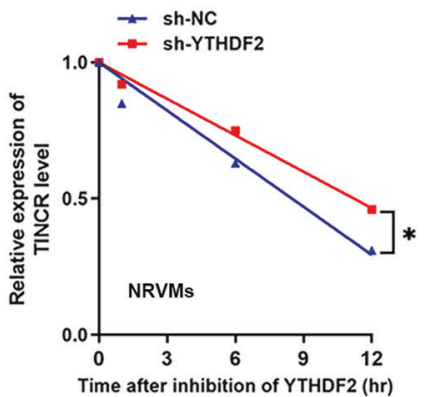

Fig. 6 TINCR was downregulated due to the METTL14-mediated m6A methylation. A Predicted binding sites of m6A modification at TINCR sequence according to the online SRAMP database (http://www.cuilab.cn/sramp). B METTL14-overexpression virus and sh-WTAP vector was injected into DCM rats. Protein band intensity of METTL14 and WTAP were quantified by western blot assay. C TINCR expression was determined in rats injected with Lv-METTL14 and(or) sh-WATP, $* * P<0.01$. D Methylated TINCR level was detected via RIP assay, and a significant downregulation by silence of WTAP and METTL14 was observed, ${ }^{* *} P<0.01,{ }^{* *} P<0.001$. E RIP assay using YTHDF2 antibody revealed a significant association with TINCR, ${ }^{* *} P<0.001$. F RNA pulldown experiment showed that YTHDF2 was enriched by TINCR probe. G Western blot validation of YTHDF2 protein following the transfection of sh-YTHDF2 vector. $\mathbf{H}$ Two cardiomyocyte cell lines were treated with AtcD, then existing TINCR was detected. Silence of YTHDF2 significantly increased degradation of TINCR, ${ }^{*} P<0.05$.

TINCR and NLRP3 expression. Mechanistically, METTL14 mediated m6A modification of TINCR, and thus suppressed TINCR expression and the NLRP3 stability, preventing the occurrence of pyroptosis.

DCM is characterized by structural and functional disorders, including myocardial cell death, myocardial fibroblast activation, left ventricular dysfunction, and metabolic deregulation [19]. Pyroptosis is characterized by programmed cell death inflammation and has been proved crucial for controlling microbial infections [20]. Accumulating evidence suggests that pyroptosis may contribute to a range of diseases, including autoimmune diseases, diabetes mellitus, nervous system-related diseases, and cardiovascular diseases [21-23]. Several reports showed that pyroptosis was closely associated with DCM progression [24]. Moreover, HG treatment could lead to the produce of active oxygen species, resulting in inflammation accompanied with elevated expression of cleaved caspase-1, IL-1 $\beta$ and IL-18 [25]. In addition, the activation of the inflammasome and the release of cytokines can promote the deposition of collagens and fibrotic formation, further exacerbating the severity of DCM [26]. Our study further confirmed that pyroptosis was involved in DCM both in vitro and in vivo. Moreover, this regulation is mainly through the regulation of NLRP3 inflammasome.

The m6A modification is deposited to RNAs by the m6A methyltransferase (writer) complex, a protein complex formed by METTL3/METTL14 heterodimeric catalytic core and a regulatory subunit, WTAP. METTL3 and METTL14 are co-located in nuclear spots and form stable complexes in a 1:1 ratio [27]. METTL14 is a pseudo-methyltransferase that stabilizes METTL3 and recognizes target RNA [28]. Here is emerging evidence to indicate that m6A modification is closely related to the occurrence and progression of CVDs, including cardiac hypertrophy, heart failure, ischemic heart disease, etc. Dorn et al. [10] demonstrated that METTL3mediated $\mathrm{m} 6 \mathrm{~A}$ modification is significant for maintaining cardiac homeostasis and normal cardiac function and revealed increased m6A methylation in cardiomyocytes under hypertrophic stimulation. However, whether METTL14 is involved in cardiovascular diseases, such as DCM are largely unknown. Our study confirmed that METTL14 was downregulated in DCM models and remarkably suppressed DCM. More importantly, METTL14 inhibits pyroptosis in a NLRP3-dependent manner, which uncovered a novel 


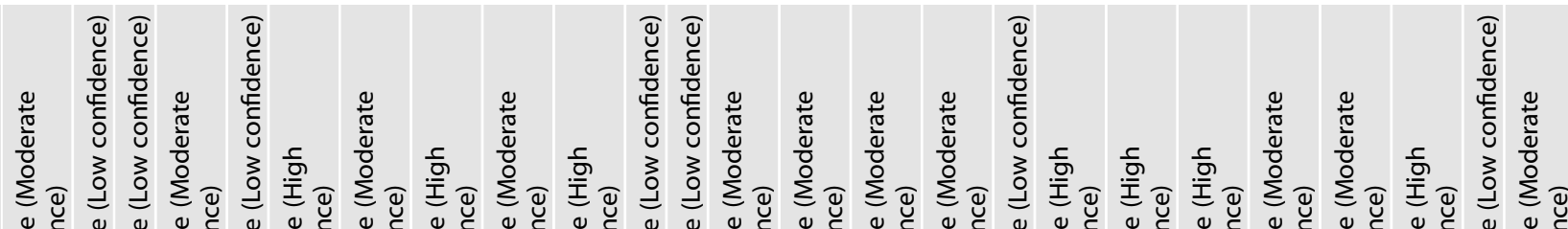

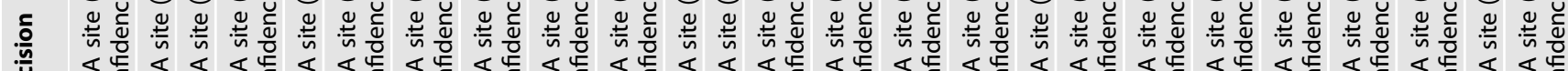

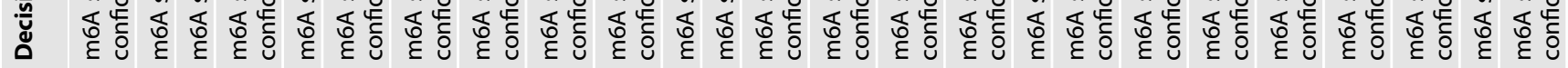

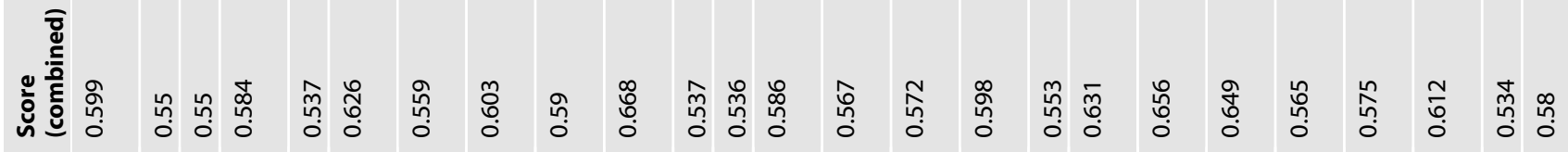

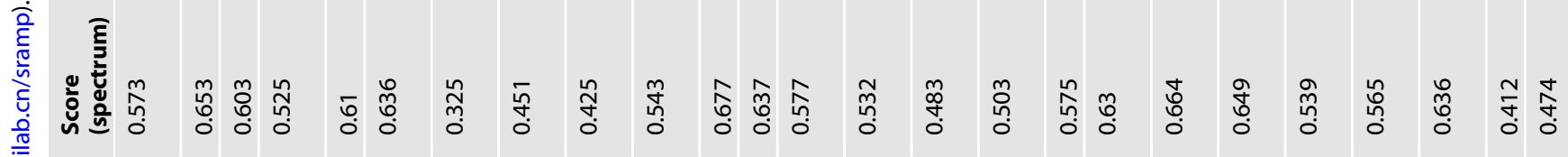
突

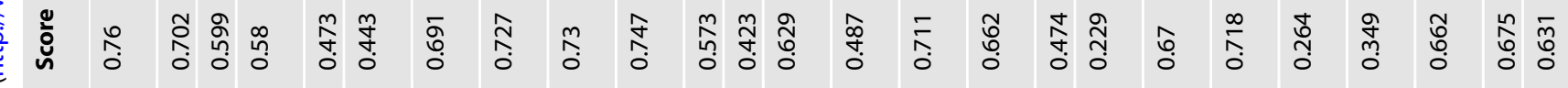

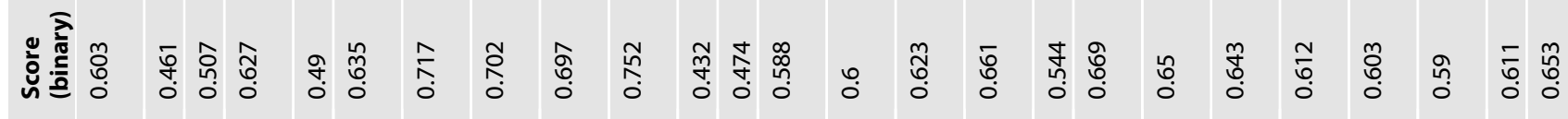

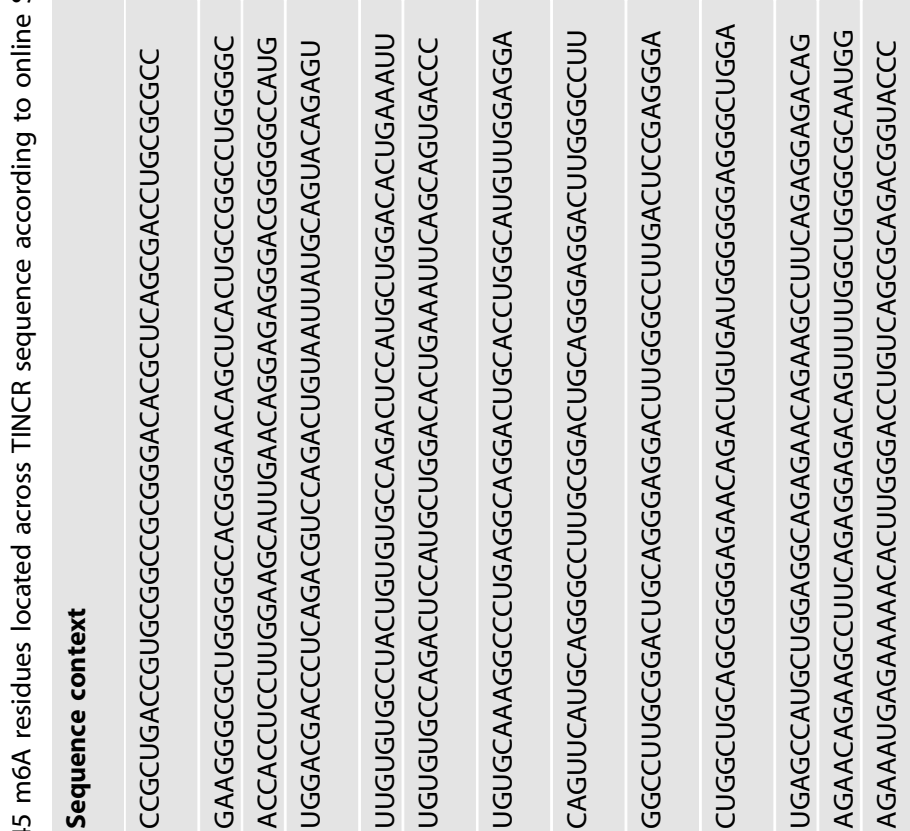

妾

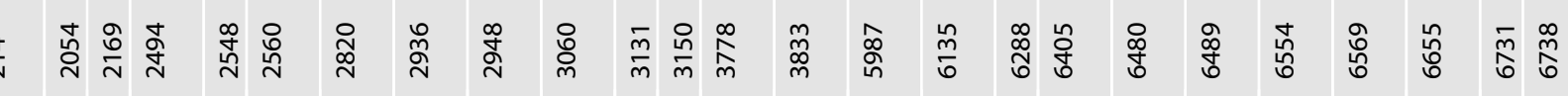
竞
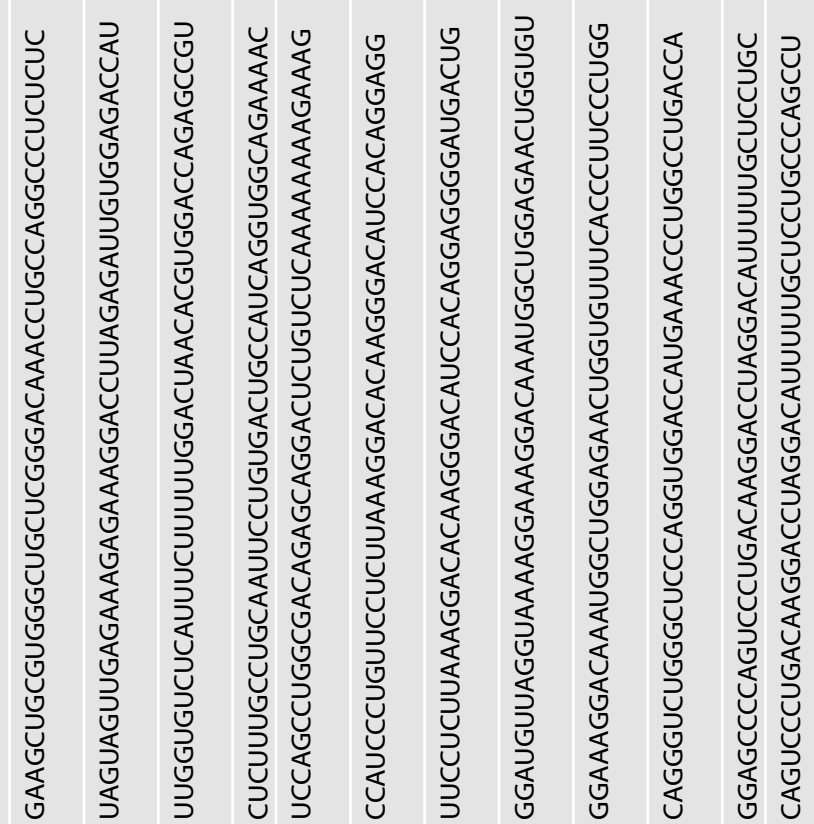


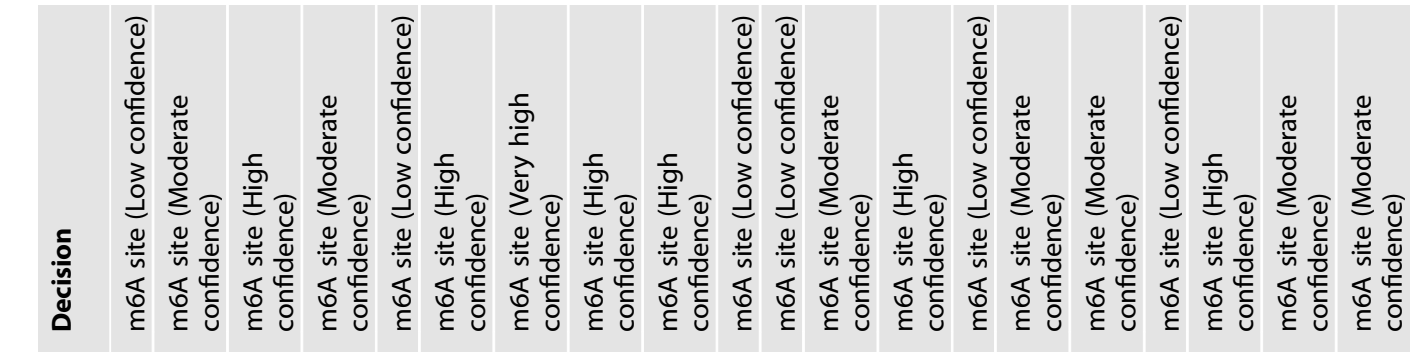

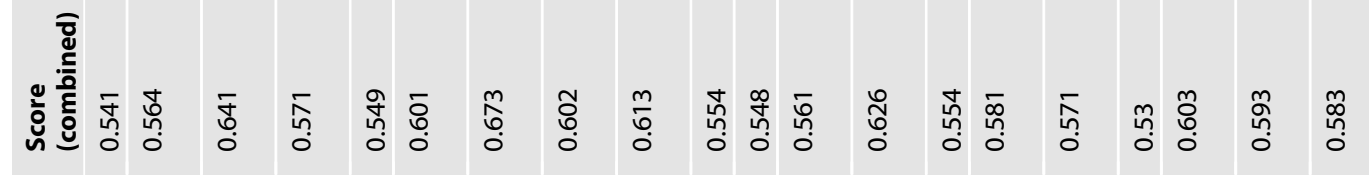

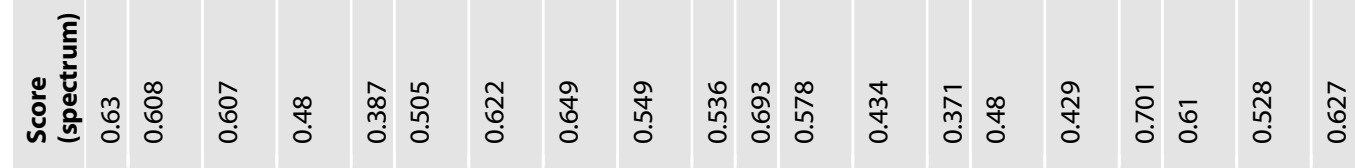

彥

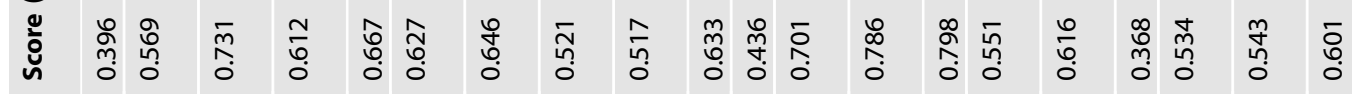

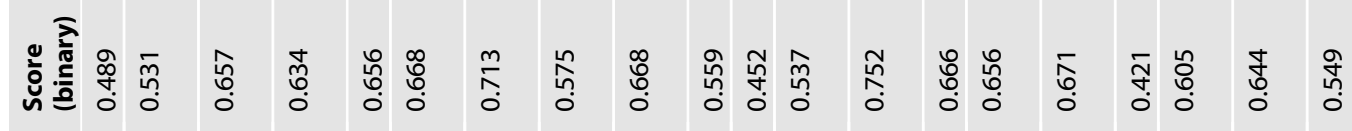

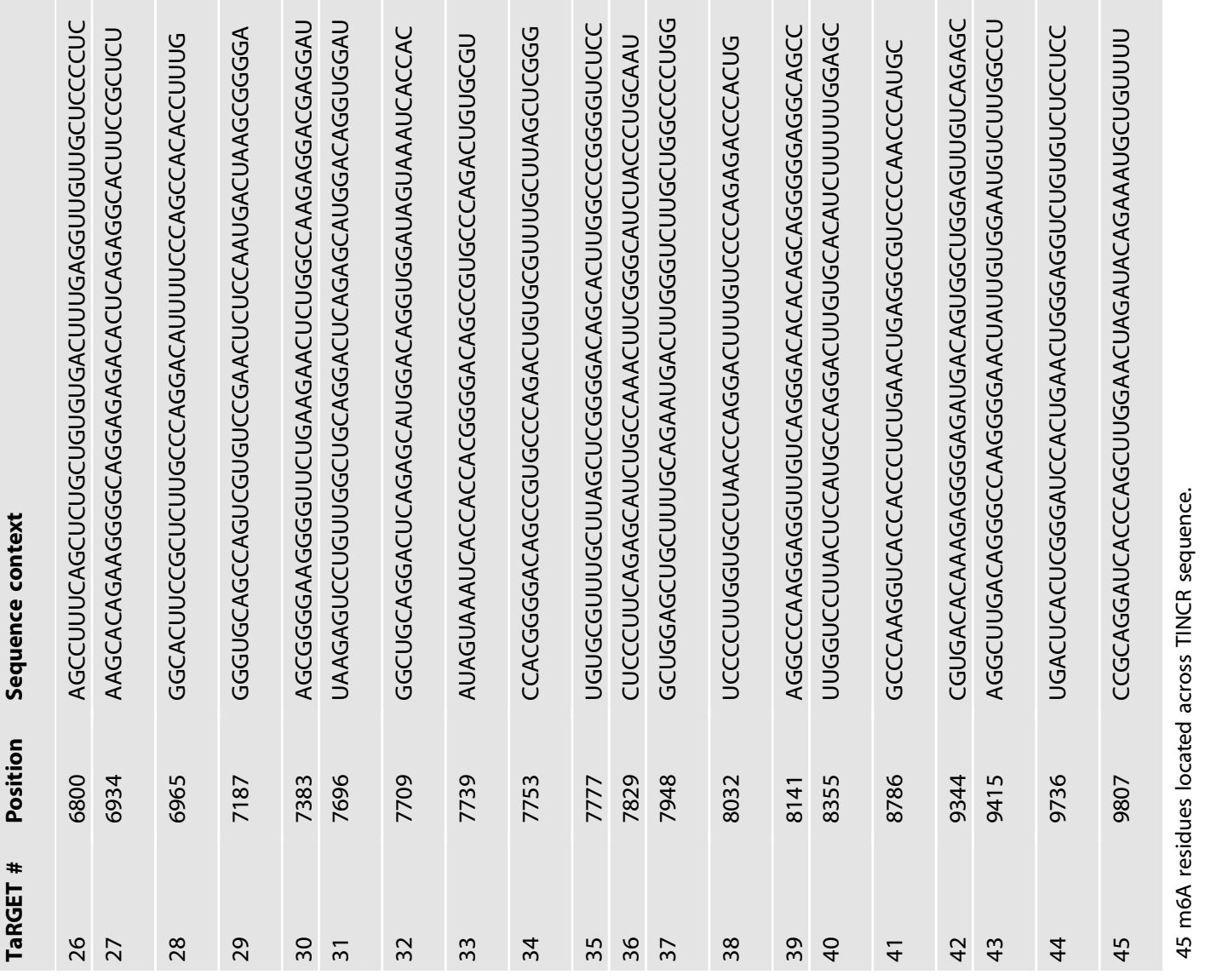



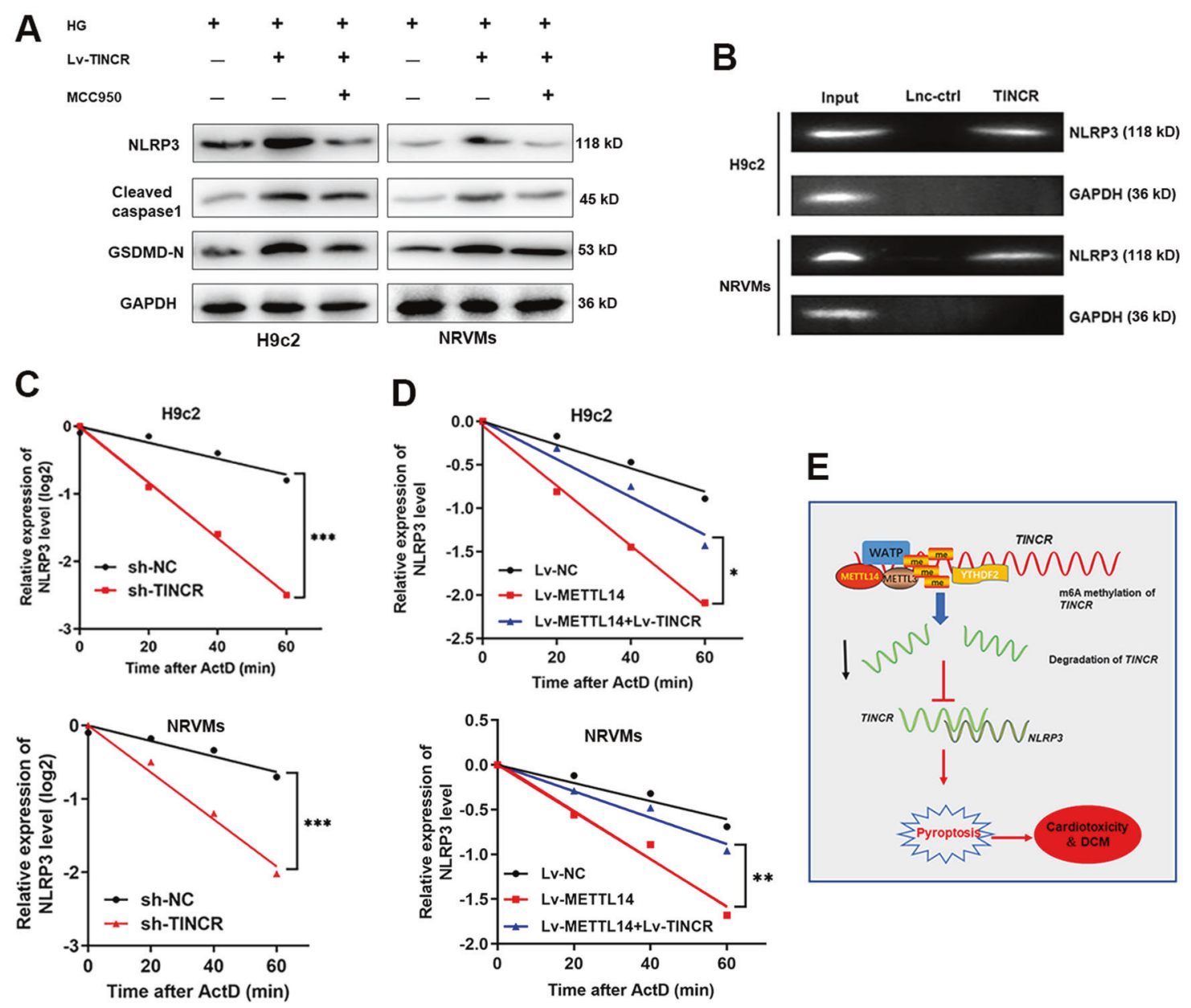

Fig. 7 TINCR functions through stabilizing NLRP3 mRNA. A Western blot experiment was performed to test the expression of pyroptosisrelated proteins in cardiomyocytes treated with LV-TINCR and MCC950. B RNA pulldown using specific TINCR probe revealed a direct interaction between TINCR and NLRP3. C Cells were treated with ActD, then existing NLRP3 mRNA was detected at different time point. Silence of TINCR significantly decreased NLRP3 stability, $P<0.001$. D METTL14 suppressed NLRP3 stability, while co-transfection of Lv-TINCR partially reversed this effect, ${ }^{*} P<0.05,{ }^{*} P<0.01$. E A scheme of the proposed mechanisms: TINCR was modified by METTL14-mediated m6A methylation, which induced suppression of TINCR in cardiomyocytes. Suppressed TINCR caused decreased stability of NLRP3 and thereby induced its downregulation. Eventually, downregulated NLRP3 inhibited pyroptosis and DCM progression.

functional role of METTL14 in pyroptosis and pyroptosis-induced DCM. Here, we need to point out that the conclusion obtained were based on the rat model induced by streptozotocin injection, which mimics the condition of type 1 diabetes. Whether the regulation of METTL14 in DCM also applies to other DCM models, such as high-fat diet model (T2DM), and what will the functional implications of METTL14 in those modes, need more strong evidence from further studies.

Recent studies have found that, in addition to the roles of $\mathrm{m} 6 \mathrm{~A}$ modifications in mRNAs, m6A modifications regulate the generation and function of noncoding RNAs, such as IncRNAs. LncRNAs are a class of transcripts more than 200 nucleotides long with no protein-coding function [29]. m6A modifications might modulate the function of IncRNAs by providing a binding site for the m6A reader proteins or by modulating the structure of the local RNA to induce RNA-binding protein entry. Previous study showed that knocking down METTL3 reduced the level of m6A modifications on specific transcripts such that the IncRNA $X$ chromosome was inactivated [30]. Gone et al. demonstrated that METTL14 mediated m6A modification suppressed LncRNA ZFAS1/ RAB22A expression and provided novel therapeutic targets for atherosclerosis [31]. Many IncRNAs show temporal differential expression, and display genic distribution in the genome. Interestingly, temporal-specific m6A-methylation with consensus m6A motif
GGACU was reported in the last exon in most IncRNAs, indicating the potential way of interaction between m6A-methylation and IncRNA expression [32]. Our former study revealed the essential role of IncRNA TINCR in pyroptosis and myocardial damage. With the consistent functions between METTL14 and TINCR, we investigated the potential regulatory mode of METTL14 and TINCR. By predicting with an online software, we uncovered several potential binding sites of m6A. RIP assay further confirmed the direct interaction.

The current hypothesis suggests that for m6A modification to exert its biological functions, it must first be recognized by $\mathrm{m} 6 \mathrm{~A}$ reader proteins. Human YTH domain family proteins include three members, YTHDF1-3, each of which comprises a highly conserved single-stranded RNA-binding domain located at the carboxy terminus (the YTH domain) and a less conserved amino-terminal region [33]. The fates of m6A modified mRNAs are dependent on m6A selective binding proteins [34]. YTHDF2 is the first identified and well-studied functional m6A-binding protein that mainly regulates the stability of mRNA [35]. YTHDF2 recognizes and binds to m6A sites in 3'UTR of mRNA through its C-terminal YTH domain to accelerate the degradation of target mRNAs [36]. As METTL14 decreased the expression of TINCR, we supposed the m6A reader could be YTHDF2 and finally, this hypothesis was confirmed, which further strengthen our previous report. 
The regulation of NLRP3-based pyroptosis by TINCR has been well defined in DOX-induced myocardial damage in our previous study. Importantly, the regulation mode also applies to DCM. This commonly used pathway in pyroptosis suggests a tight correlation between TINCR-NLRP3 pathway and pyroptosis regardless of the causes of pyroptosis. However, the detailed mechanism by which DCM/HG-induced higher expression of TINCR is not well known, which is the limitation of this study. We will follow this study and continue exploring the underlying pathways. In addition, the expression of METTL14 in a healthy population and in different organ of diabetic patients with/without organ damage could be an interesting topic in the area of diabetics. We will focus on this issue and keep exploring the regulatory mechanism of METTL14mediated pyroptosis in diabetic patients to identify its potential clinical value for predicting organ damage.

In conclusion, we demonstrated that METTL14 suppresses pyroptosis and DCM progression via m6A methylation of TINCR mRNA in an NLRP3-dependent manner. Our study not only help better understand the regulatory mechanism of METTL14mediated m6A modification in myocardial damage but is also useful for finding promising drug targets and developing novel therapeutic strategies to overcome DCM.

\section{DATA AVAILABILITY}

The analyzed data sets generated during the study are available from the corresponding author on reasonable request.

\section{REFERENCES}

1. Levelt E, Gulsin G, Neubauer S, McCann GP. MECHANISMS IN ENDOCRINOLOGY: diabetic cardiomyopathy: pathophysiology and potential metabolic interventions state of the art review. Eur J Endocrinol. 2018;178:R127-R139. https://doi.org/ 10.1530/EJE-17-0724

2. Murtaza G, Virk HUH, Khalid M, Lavie CJ, Ventura H, Mukherjee D, et al. Diabetic cardiomyopathy - A comprehensive updated review. Prog Cardiovasc Dis. 2019;62:315-26. https://doi.org/10.1016/j.pcad.2019.03.003

3. Nirengi S, Peres Valgas da Silva C, Stanford KI. Disruption of energy utilization in diabetic cardiomyopathy; a mini review. Curr Opin Pharm. 2020;54:82-90. https:// doi.org/10.1016/j.coph.2020.08.015

4. Liu X, Zhang Z, Ruan J, Pan Y, Magupalli VG, Wu H, et al. Inflammasome-activated gasdermin $D$ causes pyroptosis by forming membrane pores. Nature. 2016;535:153-8. https://doi.org/10.1038/nature18629

5. Broz P. Immunology: Caspase target drives pyroptosis. Nature. 2015;526:642-3. https://doi.org/10.1038/nature15632

6. Yang F, Li A, Qin Y, Che H, Wang Y, Lv J, et al. A novel circular RNA mediates pyroptosis of diabetic cardiomyopathy by functioning as a competing endogenous RNA. Mol Ther Nucleic Acids. 2019;17:636-43. https://doi.org/10.1016/j. omtn.2019.06.026

7. Xie $Y$, Huang $Y$, Ling $X$, Qin $H$, Wang $M$, Luo B. Chemerin/CMKLR1 axis promotes inflammation and pyroptosis by activating NLRP3 inflammasome in diabetic cardiomyopathy rat. Front Physiol. 2020;11:381 https://doi.org/10.3389/ fphys.2020.00381

8. Cao R, Fang D, Wang J, Yu Y, Ye H, Kang P, et al. ALDH2 overexpression alleviates high glucose-induced cardiotoxicity by inhibiting NLRP3 inflammasome activation. J Diabetes Res. 2019;2019:4857921 https://doi.org/10.1155/2019/4857921

9. Liu K, Gao Y, Gan K, Wu Y, Xu B, Zhang L, et al. Prognostic roles of N6methyladenosine METTL3 in different cancers: a system review and metaanalysis. Cancer Control. 2021;28:1073274821997455. https://doi.org/10.1177/ 1073274821997455

10. Qin Y, Li L, Luo E, Hou J, Yan G, Wang D, et al. Role of m6A RNA methylation in cardiovascular disease (Review). Int J Mol Med. 2020;46:1958-72. https://doi.org/ 10.3892/ijmm.2020.4746

11. Zhang $\mathrm{C}, \mathrm{Fu}$ J, Zhou $\mathrm{Y}$. A review in research progress concerning m6A methylation and immunoregulation. Front Immunol. 2019;10:922. https://doi.org/ 10.3389/fimmu.2019.00922

12. Zhang BY, Han L, Tang YF, Zhang GX, Fan XL, Zhang JJ, et al. METTL14 regulates M6A methylation-modified primary miR-19a to promote cardiovascular endothelial cell proliferation and invasion. Eur Rev Med Pharm Sci. 2020;24:7015-23. https://doi.org/10.26355/eurrev_202006_21694

13. Meng L, Lin H, Zhang J, Lin N, Sun Z, Gao F, et al. Doxorubicin induces cardiomyocyte pyroptosis via the TINCR-mediated posttranscriptional stabilization of
NLR family pyrin domain containing 3. J Mol Cell Cardiol. 2019;136:15-26. https:// doi.org/10.1016/j.yjmcc.2019.08.009

14. Liu H, Xu Y, Yao B, Sui T, Lai L, Li Z. A novel N6-methyladenosine (m6A)dependent fate decision for the IncRNA THOR. Cell Death Dis. 2020;11:613 https://doi.org/10.1038/s41419-020-02833-y

15. Jin D, Guo J, Wu Y, Yang L, Wang X, Du J, et al. m(6)A demethylase ALKBH5 inhibits tumor growth and metastasis by reducing YTHDFs-mediated YAP expression and inhibiting miR-107/LATS2-mediated YAP activity in NSCLC. Mol Cancer. 2020;19:40. https://doi.org/10.1186/s12943-020-01161-1

16. Yang $X$, Zhang $S$, He $C$, Xue $P$, Zhang L, He Z, et al. METTL14 suppresses proliferation and metastasis of colorectal cancer by down-regulating oncogenic long non-coding RNA XIST. Mol Cancer. 2020;19:46. https://doi.org/10.1186/s12943020-1146-4

17. Tarquini R, Pala L, Brancati S, Vannini G, De Cosmo S, Mazzoccoli G, et al. Clinical approach to diabetic cardiomyopathy: a review of human studies. Curr Med Chem. 2018;25:1510-24. https://doi.org/10.2174/0929867324666170705111356

18. Shaher F, Qiu H, Wang S, Hu Y, Wang W, Zhang Y, et al. Associated targets of the antioxidant cardioprotection of ganoderma lucidum in diabetic cardiomyopathy by using open targets platform: a systematic review. Biomed Res Int. 2020;2020:7136075. https://doi.org/10.1155/2020/7136075

19. Adeghate E. Molecular and cellular basis of the aetiology and management of diabetic cardiomyopathy: a short review. Mol Cell Biochem. 2004;261:187-91. https://doi.org/10.1023/b:mcbi.0000028755.86521.11

20. Xia X, Wang $X$, Zheng $Y$, Jiang J, Hu J. What role does pyroptosis play in microbial infection? J Cell Physiol. 2019;234:7885-92. https://doi.org/10.1002/jcp.27909

21. Wan, T, Li, X \& Li, Y The role of TRIM family proteins in autophagy, pyroptosis, and diabetes mellitus. Cell Biol Int, 2021. https://doi.org/10.1002/cbin.11550

22. Wu, J, Sun, J \& Meng, X Pyroptosis by caspase-11 inflammasome-Gasdermin D pathway in autoimmune diseases. Pharmacol Res, 2021;105408, https://doi.org/ 10.1016/j.phrs.2020.105408.

23. McKenzie BA, Dixit VM, Power C. Fiery cell death: pyroptosis in the central nervous system. Trends Neurosci. 2020;43:55-73. https://doi.org/10.1016/j. tins.2019.11.005

24. Xu Y, Fang $H, X u$ Q, Xu C, Yang L, Huang C. LncRNA GAS5 inhibits NLRP3 inflammasome activation-mediated pyroptosis in diabetic cardiomyopathy by targeting miR-34b-3p/AHR. Cell Cycle. 2020;19:3054-65. https://doi.org/10.1080/ 15384101.2020 .1831245

25. Gan J, Huang M, Lan G, Liu L, Xu F. High glucose induces the loss of retinal pericytes partly via NLRP3-Caspase-1-GSDMD-mediated pyroptosis. Biomed Res Int. 2020;2020:4510628. https://doi.org/10.1155/2020/4510628

26. Fuentes-Antras J, loan AM, Tunon J, Egido J, Lorenzo O. Activation of toll-like receptors and inflammasome complexes in the diabetic cardiomyopathyassociated inflammation. Int J Endocrinol. 2014;2014:847827. https://doi.org/ $10.1155 / 2014 / 847827$

27. Wang P, Doxtader KA, Nam Y. Structural basis for cooperative function of Mettl3 and Mettl14 methyltransferases. Mol Cell. 2016;63:306-17. https://doi.org/ 10.1016/j.molcel.2016.05.041

28. Weng $H$, Huang $H$, Wu $H$, Qin $X$, Zhao BS, Dong $L$, et al. METTL14 inhibits hematopoietic stem/progenitor differentiation and promotes leukemogenesis via mRNA m(6)A modification. Cell Stem Cell. 2018;22:191-205. https://doi.org/ 10.1016/j.stem.2017.11.016. e199

29. Tao L, Yang L, Huang X, Hua F, Yang X. Reconstruction and analysis of the IncRNA-miRNA-mRNA network based on competitive endogenous RNA reveal functional IncRNAs in dilated cardiomyopathy. Front Genet. 2019;10:1149. https://doi.org/10.3389/fgene.2019.01149

30. Patil DP, Chen CK, Pickering BF, Chow A, Jackson C, Guttman M, et al. m(6)A RNA methylation promotes XIST-mediated transcriptional repression. Nature. 2016;537:369-73. https://doi.org/10.1038/nature19342

31. Gong C, Fan Y, Liu J. METTL14 mediated m6A modification to LncRNA ZFAS1/ RAB22A: a novel therapeutic target for atherosclerosis. Int J Cardiol. 2021;328:177. https://doi.org/10.1016/j.ijcard.2020.12.002

32. Nie, Y, Tian, GG, Zhang, L, Lee, T, Zhang, Z, Li, J et al. Identifying cortical specific long noncoding RNAs modified by $\mathrm{m}(6) \mathrm{A}$ RNA methylation in mouse brains. Epigenetics, 2020;1-17 https://doi.org/10.1080/15592294.2020.1861170

33. Zhang Z, Theler D, Kaminska KH, Hiller M, de la Grange P, Pudimat R, et al. The YTH domain is a novel RNA binding domain. J Biol Chem. 2010;285:14701-10. https://doi.org/10.1074/jbc.M110.104711

34. Meyer KD, Jaffrey SR. Rethinking m(6)A readers, writers, and erasers. Annu Rev Cell Dev Biol. 2017;33:319-42. https://doi.org/10.1146/annurev-cellbio-100616-060758

35. Wang X, Lu Z, Gomez A, Hon GC, Yue Y, Han D, et al. N6-methyladenosinedependent regulation of messenger RNA stability. Nature. 2014;505:117-20. https://doi.org/10.1038/nature12730

36. Zhu T, Roundtree IA, Wang P, Wang X, Wang L, Sun C, et al. Crystal structure of the YTH domain of YTHDF2 reveals mechanism for recognition of N6methyladenosine. Cell Res. 2014;24:1493-6. https://doi.org/10.1038/cr.2014.152 


\section{AUTHOR CONTRIBUTIONS}

$\mathrm{LM}, \mathrm{HL}, \mathrm{FP}$ and SW mainly designed and did the research. $\mathrm{XH}$ and JW collected and analyzed the data.

\section{FUNDING}

This study is supported by the National Natural Science Foundation of China (No. 82000252, 81900345); Project From Health Department of Zhejiang Provincial (2021RC032); Medical and Health Science and Technology Plan Project of Shaoxing City (2020A13018).

\section{ETHICS APPROVAL}

The study protocol was approved by the Research Ethics Committee of Shaoxing People's Hospital, Shaoxing hospital of Zhejiang University.

\section{COMPETING INTERESTS}

The authors declare no competing interests.

\section{CONSENT FOR PUBLICATION}

Written informed consent for publication was obtained from all participants.

\section{ADDITIONAL INFORMATION}

Supplementary information The online version contains supplementary material available at https://doi.org/10.1038/s41419-021-04484-z.

Correspondence and requests for materials should be addressed to Fang Peng or Shengjie Wu.

Reprints and permission information is available at http://www.nature.com/ reprints

Publisher's note Springer Nature remains neutral with regard to jurisdictional claims in published maps and institutional affiliations.

(i) Open Access This article is licensed under a Creative Commons Attribution 4.0 International License, which permits use, sharing, adaptation, distribution and reproduction in any medium or format, as long as you give appropriate credit to the original author(s) and the source, provide a link to the Creative Commons license, and indicate if changes were made. The images or other third party material in this article are included in the article's Creative Commons license, unless indicated otherwise in a credit line to the material. If material is not included in the article's Creative Commons license and your intended use is not permitted by statutory regulation or exceeds the permitted use, you will need to obtain permission directly from the copyright holder. To view a copy of this license, visit http://creativecommons. org/licenses/by/4.0/.

(c) The Author(s) 2021 